\title{
Millimeter Wave Fifth Generation (5G) Antenna for Smartphone Application
}

\author{
Fatin Iswani Azmi ${ }^{1}$, Farid Zubir *, Mohamad Kamal A. Rahim, and Norsaidah Muhamad Nadzir
}

Advanced RF and Microwave Research Group (ARFMRG), Communication Engineering Department, School of Electrical Engineering, Faculty of Engineering, Universiti Teknologi Malaysia, 81310 UTM Johor Bahru, Johor.

*Corresponding author: faridzubir@utm.my, Tel: 607-5557252

\begin{abstract}
In this paper, a single element antenna is designed at millimeter-wave frequency bands for future 5G smartphone applications. The configuration of proposed antenna is multiple L-slots on the ground plane which is designed on a low cost FR4 board. The antenna covers a frequency range between 28 to $35 \mathrm{GHz}$ with a higher bandwidth $4.7 \mathrm{GHz}$. The antenna shows an excellent performance when integrated with the mobile phone application. The single element antenna exhibits a maximum radiation pattern around $5.945 \mathrm{dBi}$.
\end{abstract}

Keywords: 5G, single antenna, millimeter wave, Planar Inverted F-Antenna (PIFA), return loss.

C 2019 Penerbit UTM Press. All rights reserved

Article History: received 30 July 2019; accepted 20 August 2019; published 31 August 2019.

\section{INTRODUCTION}

Currently, the development of mobile phone device has progress rapidly with variety of services and application offered to users influence by the consumer needs of smaller size mobile phone with good signal performance. Therefore, mobile traffic is expected to increases on the order 1000 times compared to what is experienced today [1]. Next generation mobile network are expected to achieve 1000-fold capacity increase compared to the current generation to meet the requirements of the dramatic traffic growth [2]. According to the Cisco report of yearly visual network index (VNI), the hugely of data which are driven by smartphone, tablets and video streaming will be continuously increasing by consumers.

The fifth generation $(5 \mathrm{G})$ will be considered for the next generation that contains a very huge carrier enormous bandwidth, extreme base station and new number of antennas [3]. Due to the high demand in mobile communication, a bandwidth is concerned to increase the capacity of higher data rate to meet the consumer's requirement [4].

The design of antenna for $5 \mathrm{G}$ millimeter wave in smartphone application is quiet a challenging task to achieve excellent performance in terms of wider bandwidth and higher gain. Commonly, the Planar Inverted-F Antenna (PIFA) type is used in mobile phone application [5].

\section{LITERATURE REVIEW}

PIFA that working at lower band for mobile phone application faced many challenges such as narrow bandwidth, lower gain and low data rates [6]. There are many techniques available in order to improve gain and bandwidth while maintaining the operating frequencies at the same band. Some of the techniques are changing the design antenna parameters, position of antennas in mobile phone and others. However, all these techniques showed the insignificant improvement in terms of gain and bandwidth.

From previous work, there are several research are focused on developing millimeter wave for 5G smartphone application. A novel open slot PIFA was proposed with an elliptical shape of patch at frequency 28 GHz. The wider bandwidth for array antenna has achieved $6 \mathrm{GHz}$ at 26-32 GHz. However, the gain element for single PIFA antenna for this antenna design is lower [7].

In [8], a three-element of single PIFA has been proposed in $28 \mathrm{GHz}$ band for future millimeter wave $5 \mathrm{G}$ wireless communication. The result show the proposed antenna achieved higher gain but achieved low bandwidth which is $807 \mathrm{MHz}$.

Besides that, the proposed multiband PIFA for $5 \mathrm{G}$ mobile communication application in [9] was invent a slot on the patch. The performance of these antenna show it achieve wider bandwidth but low gain.

Therefore, alternatively PIFA which working at lower millimeter wave band have been suggested to overcome this problem. Hence, the size of PIFA is expected to be much smaller than the previous PIFA by around $70 \%$ to $75 \%$. Having PIFA in frequency millimeter wave band saves the space inside the smartphone and gives a better performance.

Consequently, PIFA now could possibly be working in array of six to eight elements occupied in smartphone instead of working at dual or triple band. As a result, PIFA that working in array at millimeter wave band can provides higher gain and wider bandwidth as well as higher data rate. 
Therefore, PIFA at the center frequency $31.5 \mathrm{GHz}$ for fifth generation $(5 \mathrm{G})$ millimeter wave which in higher frequency band will be proposed in this project to get the higher capacity data rate as well as the speed required.

\section{METHODOLOGY}

There are three phases were involved in order to design this antenna which are design the antenna, simulate the result performance of antenna and analyze the results simulation. The design of PIFA is started with the software development by using CST Software.

Analysis parameters of antenna technique was study for design this antenna to come out with the new configuration of PIFA antenna which can provide a wider bandwidth and higher gain.

\section{CONFIGURATION OF THE DESIGN PIFA IN SMARTPHONE}

A single PIFA at centre frequency $31.5 \mathrm{GHz}$ was designed and simulated using CST software. Figure 1 shows a single PIFA from the top and back view. This antenna was design on ground plane for smartphone with length $100 \mathrm{~mm}$ X $70 \mathrm{~mm}$ which is same size as a 4.5 inch smartphone. The substrate used for printed circuit board with size $100 \mathrm{~mm} \times 70 \mathrm{~mm}$ is standard low cost FR4 epoxy with dielectric constant 4.4. The thickness of the substrate used is $1.6 \mathrm{~mm}$. The via used has diameter 0.4 $\mathrm{mm}$ and its height is $1.6 \mathrm{~mm}$.

The size of radiated patch was designed with dimension of $0.5 \lambda \times 0.3125 \lambda$ at $31.5 \mathrm{GHz}$. Besides that, the length of slots used for both is $\lambda / 4$.

The simulation result of the antenna performance include of bandwidth achieved and gain.

\section{ANALYSIS OF PARAMETERS PIFA DESIGN}

The purpose of parameters design of PIFA is to ensure that the proposed design PIFA can achieve a higher gain and higher bandwidth. Each of parameters design has been studied by forming some of design using CST Software. The simulation results of these parameters design are analyzed based on return loss S11 parameter and radiation pattern to form a proposed single PIFA design with desired design specifications

\subsection{Number of Slot}

The number of slot with the L shaped on ground plane has been introduced. At the begin, the L-slot on ground plane is design to look out of its performance before come out with double L-slots on ground plane. It can be seen in Figure 2, the L-slot is created on the ground plane for PIFA design and its simulation result of S11 parameter is shown in Figure 3.

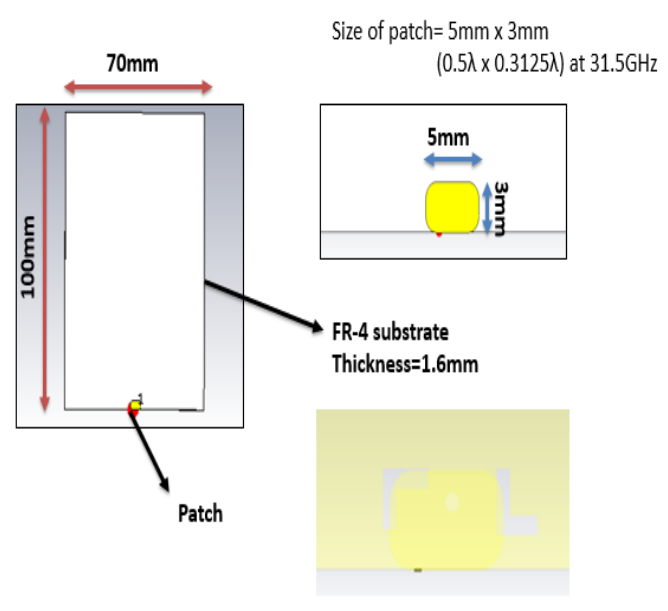

(a)

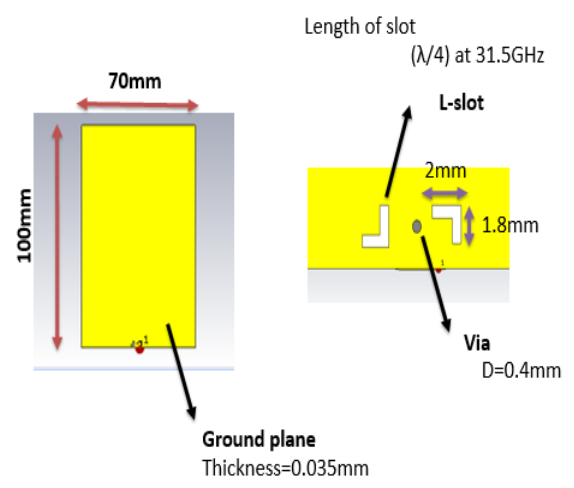

(b)
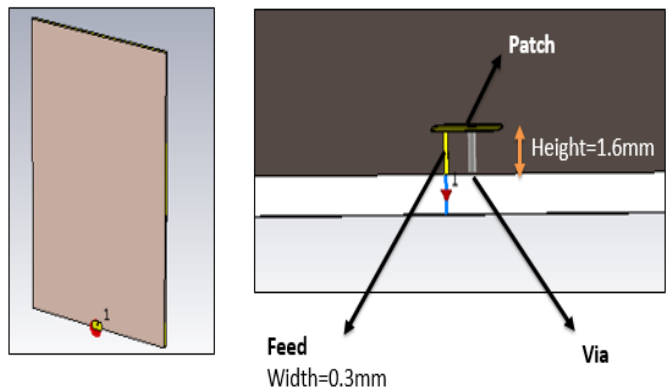

(c)

Figure 1. (a) Single proposed design PIFA (top view), (b) Single proposed design PIFA (back view), (c) Single proposed design PIFA (perspective view view)

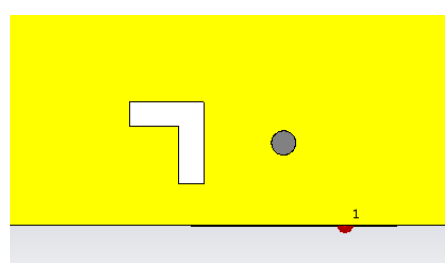

Figure 2. L-slot on ground plane (back view) 


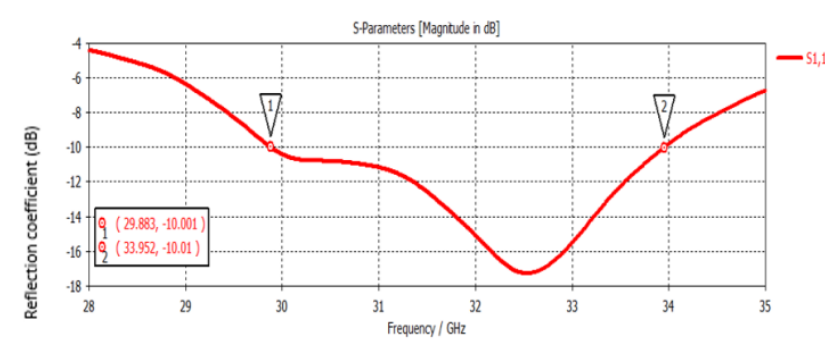

Figure 3. S11 parameter for L-slot on ground plane

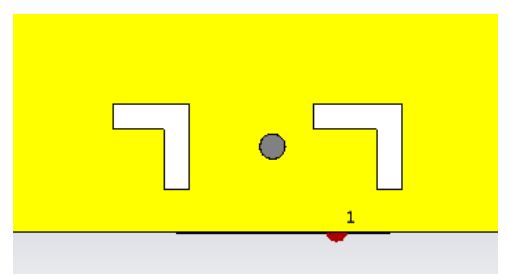

Figure 4. Two L-slots on ground plane (back view)

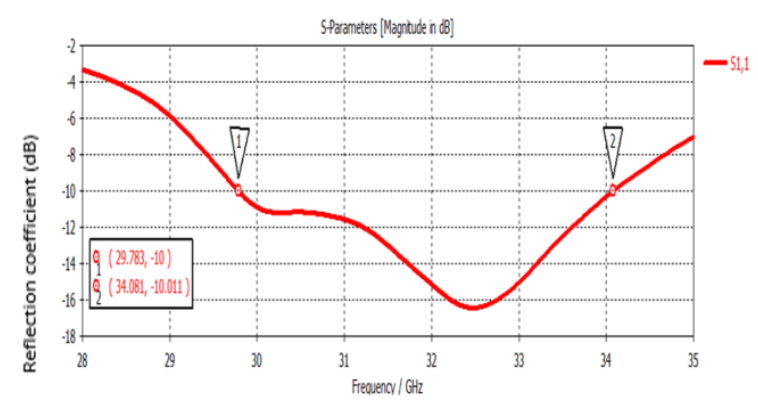

Figure 5. S11 parameter for two L-slots on ground plane

The result of S11 parameter for L-slot in Figure 3 achieve more than $10 \mathrm{~dB}$ of return loss and its bandwidth of $4.069 \mathrm{GHz}$ which ranging from $29.883 \mathrm{GHz}$ to 33.952 $\mathrm{GHz}$. Figure 4 shows two L-slots which designed on ground plane and its return loss achieved more than $10 \mathrm{~dB}$ with bandwidth of $4.298 \mathrm{GHz}$ as shown in Figure 5.

As a result, two L-slots achieved a higher bandwidth than one slot. Both designs of slots have achieved more than $-10 \mathrm{~dB}$ of return loss. However, the realized gain achieved for one slot designed is higher which is 6.159 $\mathrm{dB}$ compared to the two slots which is $5.935 \mathrm{GHz}$ at frequency $31.5 \mathrm{GHz}$.

\subsection{Position of Slots}

The two L-slots on the ground plane have been analyzed with varies positions There are five different positions for the two L-slots. These positions are shown as in Figure 6.

Figure 7 illustrates the return loss of S11 parameters for all five positions. The position slot is chosen based on the higher bandwidth and the realized gain. Position 5 achieved the highest bandwidth, thus was selected as the position to be used on two L-slots for ground plane.
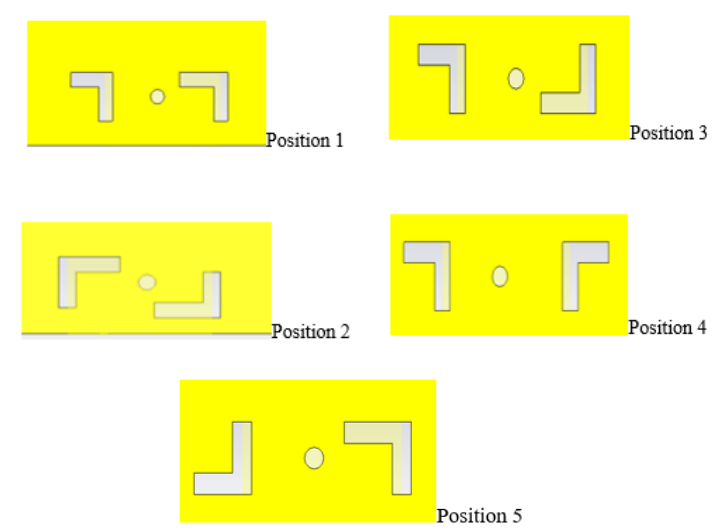

Figure 6. Two L-slots with five different position on the ground plane

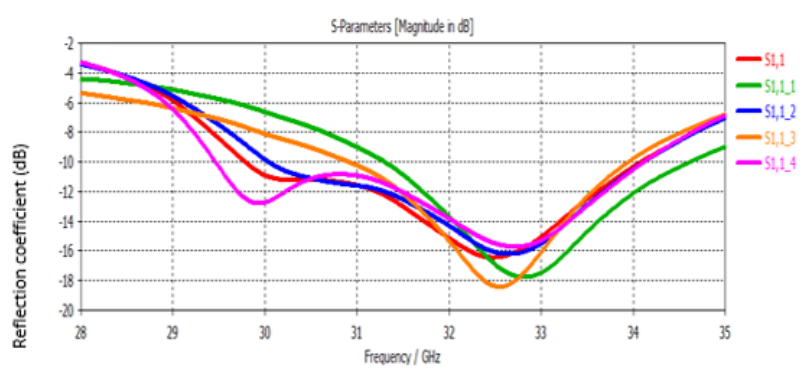

Figure 7. S11 parameter for all positions of L-slots

\subsection{Arrangement of Via}

The arrangement of via has been analyzed under three conditions; near to slot, far from slots and in between with two slots. The distance of via from the slots is the main important factor that affects the S11 parameter and radiation pattern performance. All three analysis are done within the same distance of movement of the via. The simulation results for three arrangements of via are shown in Figure 8.

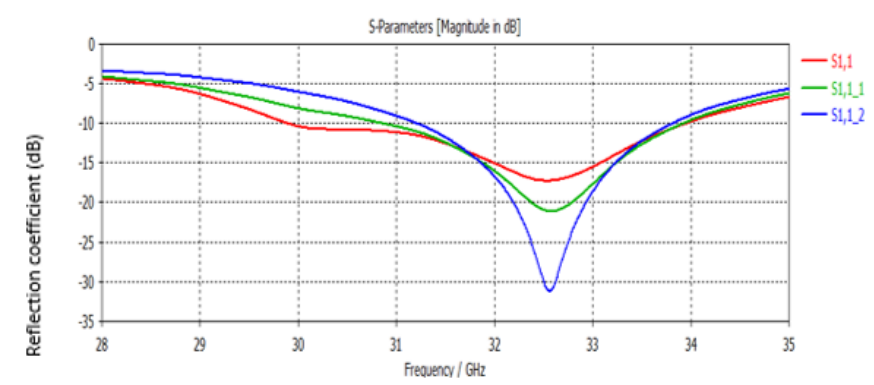

Figure 8. Combination of S11 parameter results for three different position of via

\subsection{Width of Feed}

Width of feed is another important factor in achieving wider bandwidth. The analysis for the width of feed has been varies with some different size of feed. Simulation results are shown in Figure 9. 


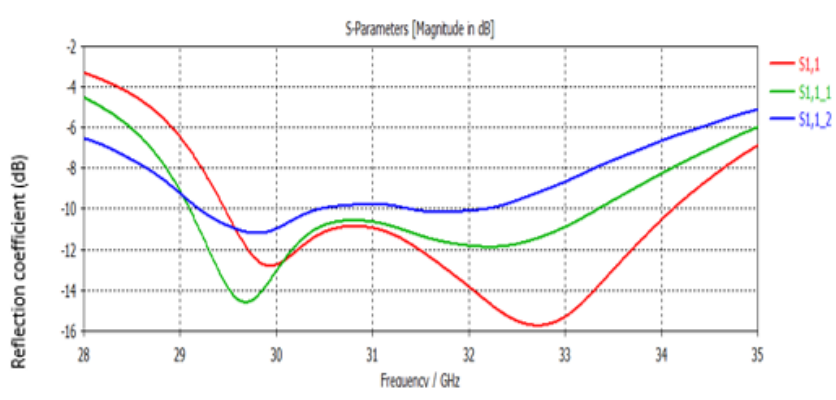

Figure 9. Combination of S11 parameter results for different width of feed

As shown in Figure 9, the return loss achieved is less than $-10 \mathrm{~dB}$ when the width size of feed increased. Furthermore, the bandwidth decreases as the gain decreased. Therefore, the width size of feed should not be too bigger to maintain the better performance of return loss and realized gain.

\subsection{Shape for Radiated patch}

There are two different shapes analyzed in this PIFA design which are rectangular and ellipse shape. These two shapes gave different results based on S11 parameter readings and realized gain radiation pattern.

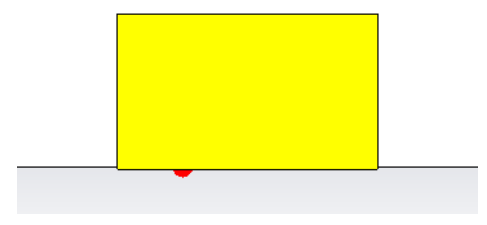

Figure 10. Rectangular patch

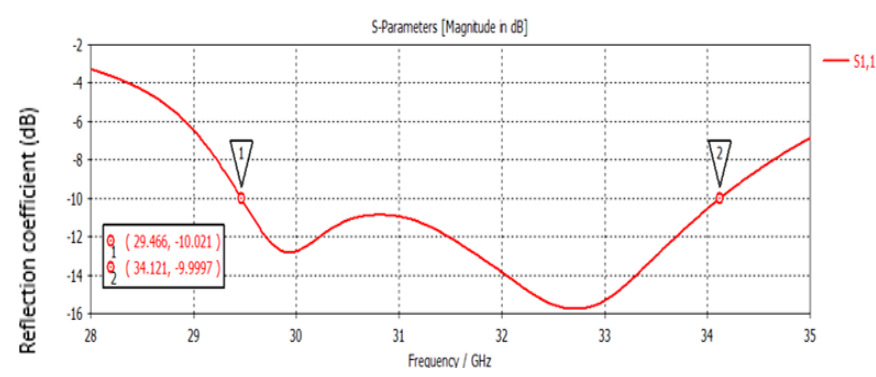

Figure 11. S11 parameter result for rectangular patch

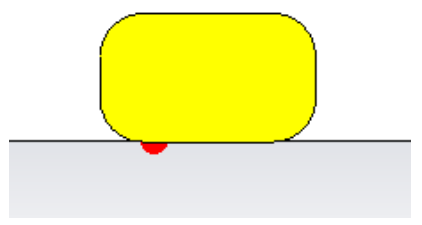

Figure 12. Ellipse patch

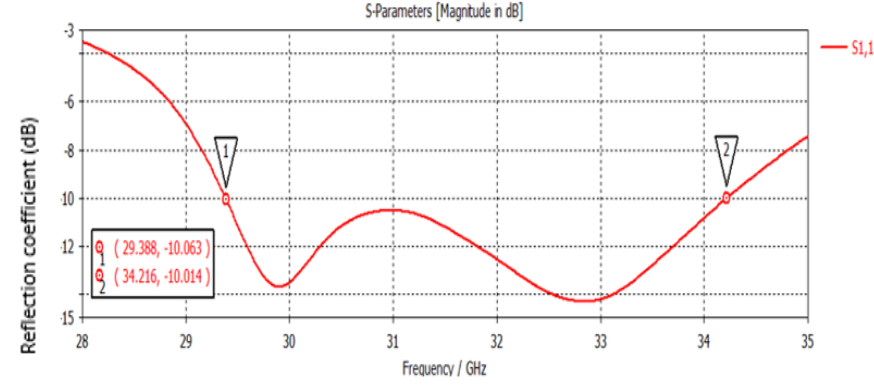

Figure 13. S11 parameter result for ellipse patch

From the simulation results shown in Figure 11 and Figure 13, the ellipse shape is wider than the rectangular shape. The achieved bandwidth for ellipse shape is 4.828 $\mathrm{GHz}$ while for rectangular shape is $4.655 \mathrm{GHz}$. Moreover, the ellipse patch shape showed an improvement on realized gain which is $4.137 \mathrm{~dB}$ while for rectangular patch achieved gain is $4.057 \mathrm{~dB}$.

\section{SINGLE PROPOSED PIFA PERFORMANCE}

The proposed single PIFA is designed based on the analysis parameters design as discussed previously. The simulation results of S11 parameter is shown in Figure 14 Achieved bandwidth for this antenna is around $4.7 \mathrm{GHz}$.

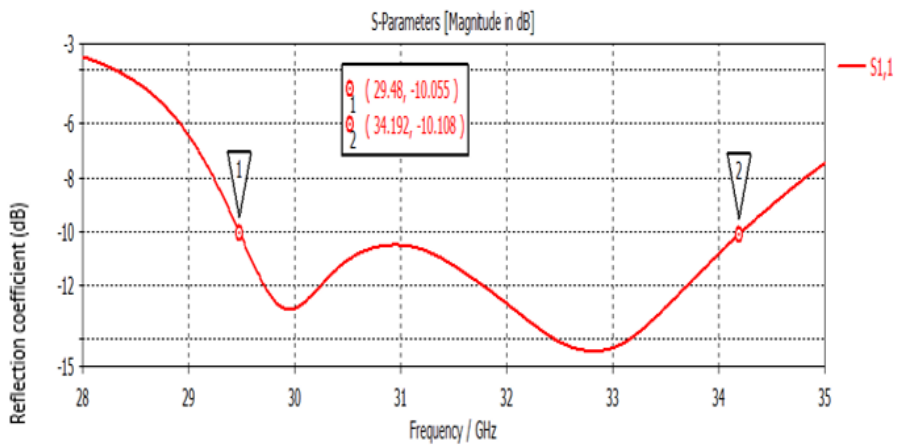

Figure 14. S11 parameter result for single proposed design PIFA

The simulated $3 \mathrm{D}$ radiation pattern of the proposed antenna element can be seen in Fig. 15 the proposed antenna produced a stable radiation pattern and gain of at least $4.137 \mathrm{~dB}$ throughtout the bands of interest.

\section{CONCLUSION}

The proposed single element PIFA at operating frequency band $28 \mathrm{GHz}$ to $35 \mathrm{GHz}$ is introduced with a new configuration of multiple L-slots on the ground plane. In order to achieve a wider bandwidth and higher gain, some of parameter designs have been studied to observe the effect changing parameters towards antenna performance. The antenna shows improvements toward the bandwidth and gain. Thus, the users can experience higher data rates.

Based on the simulation results on the proposed design single PIFA, the bandwidth achieved is quite wider which is $4.7 \mathrm{GHz}$ while the realized gain achieved is $4.137 \mathrm{~dB}$ (5.945 dBi). The next step is the antenna array element 
can be design and simulate using the proposed single element PIFA in this project to achieve a better performance for smartphone application in the future $5 \mathrm{G}$ work.

Fabrication and measurement of the design can be compared with the simulation results obtained in this project for verification purpose.

\section{REFERENCES}

[1] "Millimeter wave mobile communication for $5 \mathrm{G}$ Cellular", (Oct 23, 2014). Available from https://www.slideshare.net/raghubraghu/ppt-onmillimeter-wave.

[2] Jangeun Jun, and Mihail L. Sichitiu. (2003). 'The Nominal Capacity of Wireless Mesh Networks', Wireless Communications, IEEE, 10, 8-14.

[3] Theodore S Rappaport, James N Murdock, and Felix Gutierrez (2011). 'State of the Art in $60-\mathrm{GHz}$ Integrated Circuits and Systems for Wireless Communications', Proceedings of the IEEE, 99, 1390-436.

[4] Theodore S. Rappaport, Shu Sun, Rimma Mayzus, et. al. "Millimeter Wave Mobile Communications for
5G Cellular: It Will Work!", IEEE Access, (May 10, 2015).

[5] "Millimeter wave mobile communication for $5 \mathrm{G}$ Cellular", (Oct 23, 2014). Available from https://www.slideshare.net/raghubraghu/ppt-onmillimeter-wave.

[6] Nazem Alsmadi and Khalid Saif. "Mobile Phone Antenna Design", (May 25, 2015).

[7] Manoj Stanley, Yi Huang, Tian Loh, Qian Xu, Hanyang Wang, and Hai Zhou. (2017). 'A High Gain Steerable Millimeter-Wave Antenna Array for 5G Smartphone.

[8] Osama M. HAraz, Mohamad Asyraf and Saleh Alshebeili (2015). "Single-Band PIFA MIMO Antenna System Design for future 5G Wireless Communication Wireless", IEE 11th International Conference on Wireless and Mobile Computing, Networking and Communication (WiMob).

[9] Rahul Tiwari and Dr. H.V.Kumaraswamy, "Multiband PIFA for 5G Mobile Communication Application", International Research Journal of Engineering and Technology (IRJET). Vol.03. Issue.06. June,2016.

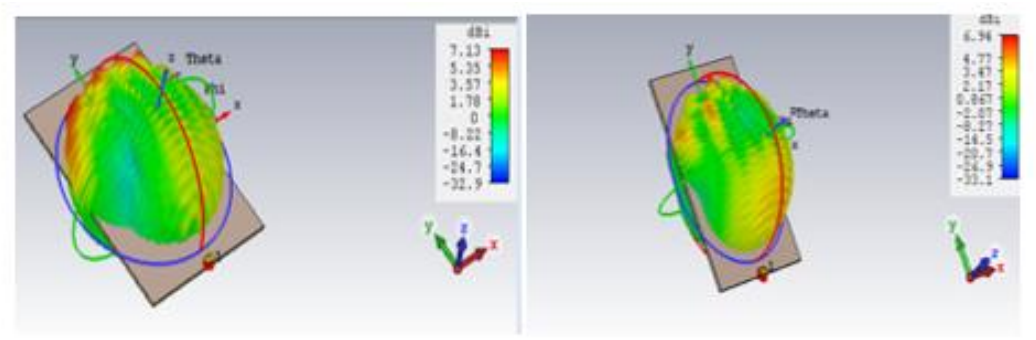

(a) $28 \mathrm{GHz}$

(b) $30 \mathrm{GHz}$

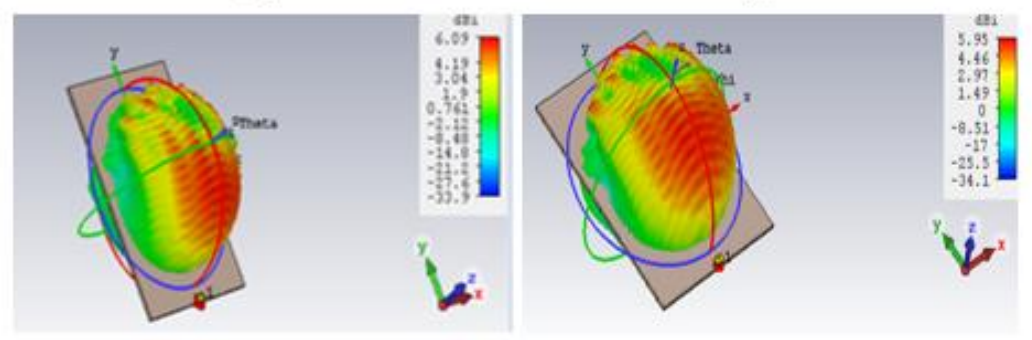

(c) $31.5 \mathrm{GHz}$

(d) $32 \mathrm{GHz}$

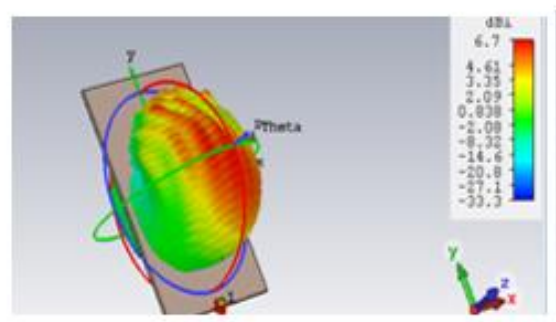

(e) $34 \mathrm{GHz}$

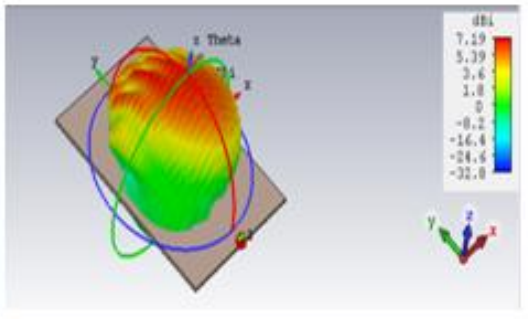

(f) $35 \mathrm{GHz}$

Figure 15. 3D radiation pattern for proposed antenna at frequencies $28 \mathrm{GHz}$, $30 \mathrm{GHz}, 31.5 \mathrm{GHz}, 32 \mathrm{GHz}, 34 \mathrm{GHz}$, and $35 \mathrm{GHz}$ 